\title{
Orientational preference and influence of rotation on methane mobility in one-dimensional channels
}

\author{
Shreyas Y. Bhide \\ Solid State and Structural Chemistry Unit, Indian Institute of Science, Bangalore-560012, India \\ S. Yashonath ${ }^{\text {a) }}$ \\ Solid State and Structural Chemistry Unit and Supercomputer Education and Research Centre, \\ Indian Institute of Science, Bangalore-560012, India
}

\begin{abstract}
Molecular dynamics investigations of methane in one-dimensional channel systems of $\mathrm{AlPO}_{4}-5$ and the carbon nanotube are reported. Methane shows contrasting orientational preference over $1+3$ and $2+2$ orientations inside $\mathrm{AlPO}_{4}-5$ and the carbon nanotube. In $\mathrm{AlPO}_{4}-5,2+2$ orientation is preferred in narrow parts of the channel while $1+3$ orientation is predominant in the wider part of the channel. In the carbon nanotube $2+2$ is preferred over $1+3$ orientation throughout the channel. Thus, the orientation of methane can yield insight into methane-host interactions. Simulations with and without allowing for rotation of methane suggest that rotation aids translational mobility of methane in both the channel systems studied. It is shown that molecules with certain orientations are associated with lower mobility, both in $\mathrm{AlPO}_{4}-5$ and carbon nanotube. Further, translational motion exhibits diffusive behavior for methane in $\mathrm{AlPO}_{4}-5$ and superdiffusive behavior in the carbon nanotube. These results suggest that particular orientation or rotation in general can strongly influence translational mobility of methane in one-dimensional channels. It is seen that levitation effects influence rotational motion in addition to the translational motion.
\end{abstract}

\section{INTRODUCTION}

In recent years there has been an increasing interest in studying properties of confined fluids in microporous solids. ${ }^{1,2}$ In particular there have been many studies directed towards understanding properties of hydrocarbons in zeolites. ${ }^{3,4}$ This is primarily due to the importance of zeolites in the petrochemical industry for cracking of long chain hydrocarbons and separation of mixtures. In both these processes mobility of molecules inside the microporous host plays a crucial role. This necessitates the study of influence of confinement on self-diffusivity of molecules.

In the case of polyatomic molecules in addition to translational degrees of freedom, rotational motion is also present. This prompts some questions: In what manner the rotational diffusivity of a molecule changes in confinement? How does the translational motion of a molecule get effected by its rotational motion in confinement? Answers to these questions can give a better understanding about the mobility of polyatomic molecules in confinement. This in turn can help in the designing of better separation procedures.

In an attempt to answer the abovementioned and other related questions we have studied methane in onedimensional channels. While methane is the simplest member of the hydrocarbon series, one-dimensional channels represent the simplest topology of interconnected pores which are devoid of complications associated with the complexities

\footnotetext{
a) Also at Condensed Matter Theory Unit, Jawaharlal Nehru Center for Advanced Scientific Research. Author to whom correspondence should be addressed.
}

of higher dimensional channels. One-dimensional channel systems studied in the present work are $\mathrm{AlPO}_{4}-5$ and the carbon nanotube. These have a varying degree of surface roughness and modulation of channel diameter along the channel. $\mathrm{AlPO}_{4}-5$ has rough or structured channel walls and it shows modulation in diameter along the channel. As opposed to this the wall of the carbon nanotube is smooth or structureless and it has a constant diameter throughout. Adsorption of methane in one-dimensional channel systems of aluminophosphates ${ }^{5}$ and carbon nanotubes ${ }^{6,7}$ has been studied experimentally and through simulations by the grand canonical Monte Carlo method. ${ }^{8,9}$ Dynamics of methane in $\mathrm{AlPO}_{4}-5$ has been studied by the pulsed field gradient (PFG) NMR technique ${ }^{10}$ which suggested the existence of single file diffusion for methane in $\mathrm{AlPO}_{4}-5$. Single file motion implies that sorbates cannot pass one another inside a channel. However, PFG-NMR (Ref. 11) studies by others as well as quasielastic neutron scattering ${ }^{12}$ (QENS) studies of methane dynamics in $\mathrm{AlPO}_{4}-5$ observed ordinary diffusive behavior suggesting thereby that sorbates can, in fact, pass each other. A plausible explanation of this difference in the observations is that the time over which the system was probed is different in the two cases. Yet another possibility is that the samples of the host materials used in different studies were slightly different. This has been pointed out by Jobic et al. ${ }^{12}$ The passing frequency of methane molecules inside $\mathrm{AlPO}_{4}-5$ has been calculated by molecular dynamics (MD) simulations. ${ }^{13}$ This study showed that methane undergoes normal diffusion inside $\mathrm{AlPO}_{4}-5$. The adsorbate passage has also been characterized by the free energy barrier experi- 
enced by methane molecules when they pass each other inside an $\mathrm{AlPO}_{4}-5$ channel. ${ }^{14} \mathrm{MD}$ study of methane in the carbon nanotube ${ }^{15}$ suggested that methane exhibits normal diffusive behavior for nanotubes having different diameters. Results from experimental and simulation studies mentioned above raise an important question: Are the results highly sensitive to slight changes in the potential parameters and channel dimension? The present work attempts to understand this by studying the dependence of orientational preference methane on channel dimension vis-à-vis potential parameters.

The present study has been carried out in the dilute limit to bring out the effect of confinement on dynamics of adsorbates in an unambiguous manner. To understand the energetics and dynamics we have calculated static potential energy along and across the channel. Also we have tried to correlate the orientation of methane with its position inside a channel. The effect of rotation of methane on translational mobility is studied by calculating mean squared displacement of methane in two cases: First, by observing mobility in simulation where both translational and rotational motion is allowed and in the second case, by freezing only rotational degrees of freedom. These properties are compared with experimentally observed values where available as well as simulation results of bulk methane.

\section{STRUCTURE OF HOSTS}

The two structures, namely, (i) $\mathrm{AlPO}_{4}-5$ and (ii) carbon nanotube have one-dimensional channels along the $c$-axis.

$\mathrm{AlPO}_{4}-5$ crystallizes in hexagonal space group $\mathrm{P} 6 / \mathrm{mcc}$ with $a=13.77$ and $c=8.38 \AA .{ }^{16}$ There are 72 atoms in one unit cell. The channel does not have a uniform diameter throughout [Figs. 1(a) and 1 (b)], it is narrow near $z \approx 0.25 c$ and $0.75 c($ diam $\approx 10.02 \AA)$ but broader in the rest of the region $($ diam $\approx 11.37 \AA)$.

The carbon nanotube structure is constructed by the method of the armchair mode of rolling the graphite sheet $^{17,18}$ [see Fig. 1(c)]. It is possible to have a carbon nanotube with different diameters. Here, we have chosen $(8,8)$ a single walled carbon nanotube which contains 640 carbon atoms. Unlike $\mathrm{AlPO}_{4}-5$ the carbon nanotube has a uniform diameter and the wall is smooth and homogeneous throughout.

Thus, these one-dimensional channels represent structures with different channel diameters and differences in surface roughness and homogeneity of channel walls.

\section{INTERMOLECULAR POTENTIAL}

Methane is modeled as a five site rigid molecule. The Lennard-Jones-type potential is considered for sorbatesorbate and sorbate-host interactions,

$$
\phi_{i j}\left(r_{i j}\right)=-\frac{A_{i j}}{r_{i j}^{6}}+\frac{B_{i j}}{r_{i j}^{12}} .
$$

The potential parameters $A_{i j}=4 \epsilon_{i j} \sigma_{i j}^{6}$ and $B_{i j}=4 \epsilon_{i j} \sigma_{i j}^{12}$ for the sorbate-sorbate interaction are taken from Murad et al. ${ }^{19}$ These are listed in Table I. Methane is assumed to

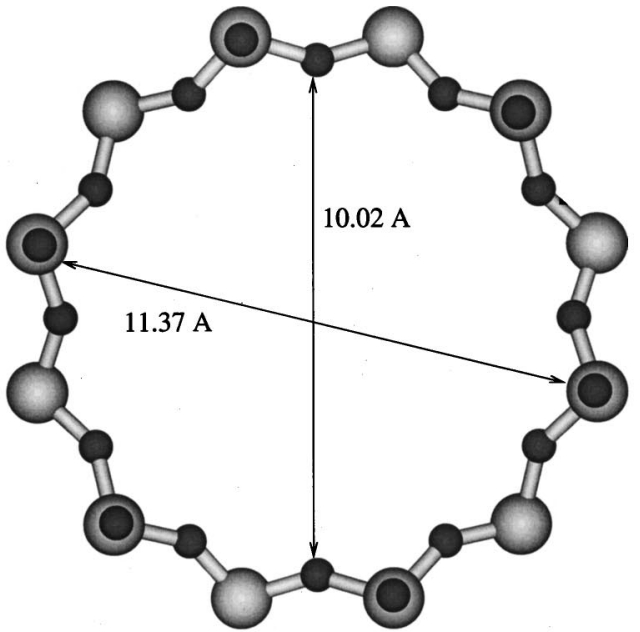

(a)

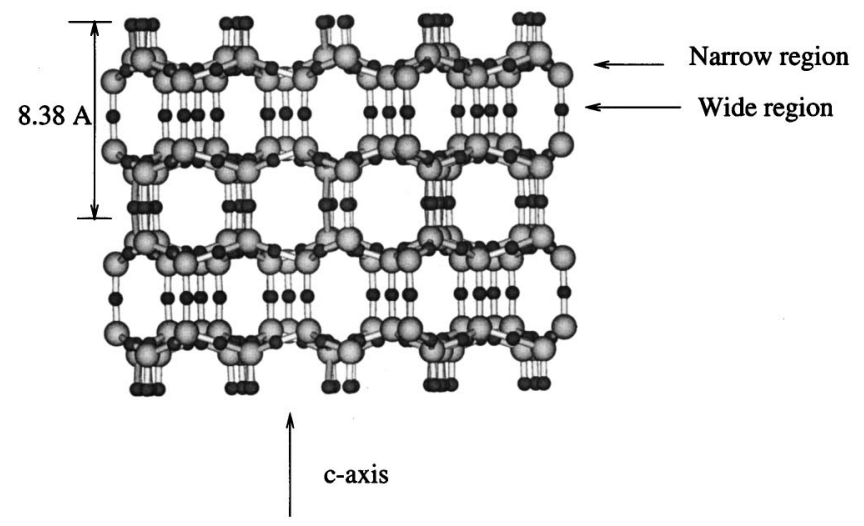

(b)

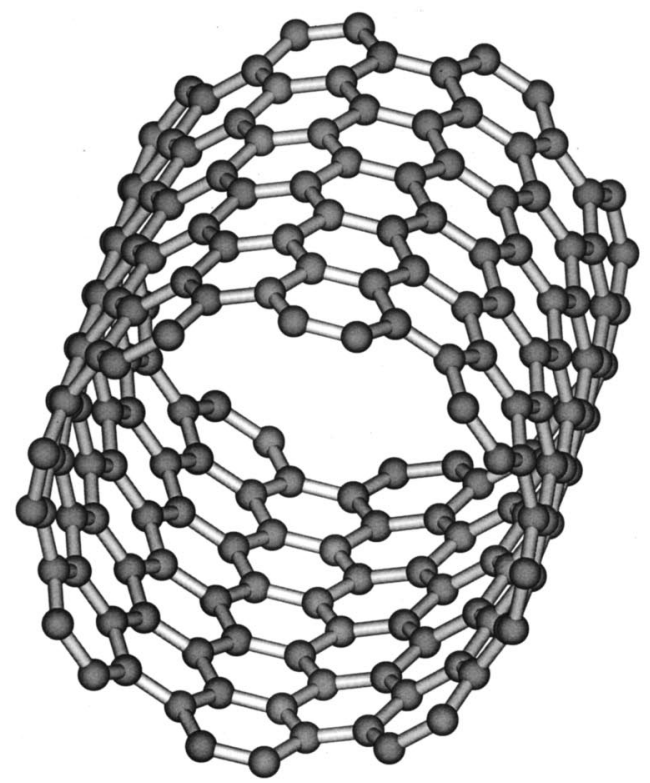

(c)

FIG. 1. Schematic view of different hosts and their channel diameters. For $\mathrm{AlPO}_{4}-5$ dark spheres correspond to oxygens. (a) A cross-sectional view of the $\mathrm{AlPO}_{4}-5$ channel. There are two diameters, the narrow $(10.02 \AA)$ and the broad $(11.37 \AA)$ which correspond, respectively, to the 12-ring window plane and region connecting two such window planes. (b) Vertical view of the $\mathrm{AlPO}_{4}-5$ channel with the different regions indicated along the channel. (c) Carbon nanotube. 
TABLE I. Potential parameters for $\mathrm{CH}_{4}-\mathrm{CH}_{4}$ and $\mathrm{CH}_{4}$-host interaction.

\begin{tabular}{lcc}
\hline \hline & $A_{i j} \times 10^{3}(\mathrm{~kJ} / \mathrm{mol}) \AA^{6}$ & $B_{i j} \times 10^{6}(\mathrm{~kJ} / \mathrm{mol}) \AA^{12}$ \\
\hline $\mathrm{C}-\mathrm{C}$ & 2.2920 & 3.2396 \\
$\mathrm{C}-\mathrm{H}$ & 0.5440 & 0.3926 \\
$\mathrm{H}-\mathrm{H}$ & 0.1354 & 0.06711 \\
AlPO -5 & & \\
$\mathrm{C}-\mathrm{O}$ & 2.0189 & 1.3024 \\
$\mathrm{H}-\mathrm{O}$ & 0.4663 & 0.1693 \\
Carbon nanotube & & \\
$\mathrm{C}-\mathrm{C}_{n}$ & 1.8161 & 2.6839 \\
$\mathrm{H}-\mathrm{C}_{n}$ & 0.4533 & 0.4074 \\
\hline \hline
\end{tabular}

interact only with the oxygen atoms of $\mathrm{AlPO}_{4}-5$. The methane-host interaction parameters for $\mathrm{AlPO}_{4}-5$ and the carbon nanotube are also listed in Table I.

\section{COMPUTATIONAL DETAILS}

All the MD simulations have been carried out in a microcanonical ensemble $(N V E)$, with cubic periodic boundary conditions. The system consists of $16 \times 16 \times 6$ unit cells in case of $\mathrm{AlPO}_{4}-5$ which makes in all 256 channels along the $c$ direction with each channel 6 unit cells long. For the carbon nanotube 256 tubes are considered arranged in a hexagonal fashion with all tubes parallel to the $c$-direction. Two channel centers are separated by a distance of $14.28 \AA$. Two methane molecules reside in each channel which corresponds to a total of 512 molecules in each host. This corresponds to a concentration of 0.33 molecule per unit cell of $\mathrm{AlPO}_{4}-5$ and 0.040 molecule/ $\AA$ inside the carbon nanotube. The methane-methane interaction is minimal and therefore the role of the methane-host interaction is dominant. The spherical cutoff radius measured from the center-of-mass of methane for both methane-methane and methane-host interaction is taken to be $12 \AA$ for aluminophosphates and for the carbon nanotube. Thus, interactions extend to molecules and host atoms in neighboring channels as well. For bulk methane, calculations are carried out at a density of $0.29 \mathrm{~g} / \mathrm{cm}^{3}$ for a total of 108 molecules. This density corresponds to bulk liquid density of methane ${ }^{19}$ which is higher than the density of methane considered in $\mathrm{AlPO}_{4}-5$ and carbon nanotube in the present study. The spherical cutoff radius is taken to be $11 \AA$ for bulk methane calculations.

The gear predictor-corrector algorithm ${ }^{20}$ is used for integration of translational and rotational equations of motion for methane molecules. For studying the dynamics of methane molecules without rotation the rotational degrees of freedom are not allowed. In this case, each molecule is assigned to a random orientation which remains the same throughout the MD run. Without rotation MD of methane is carried out in both host structures, $\mathrm{AlPO}_{4}-5$ and carbon nanotube as well as in bulk methane. The host structure is kept rigid. A time step of 1 fs has been used. This gives conservation in total energy of 5 in $10^{5}$. Different properties are calculated using positions and velocities stored at every 20 fs. The system is equilibrated for $100 \mathrm{ps}$ in each case. At the beginning of the equilibration phase, methane molecules are placed at the center of the channel with initial velocities chosen randomly from a uniform distribution. During equilibration

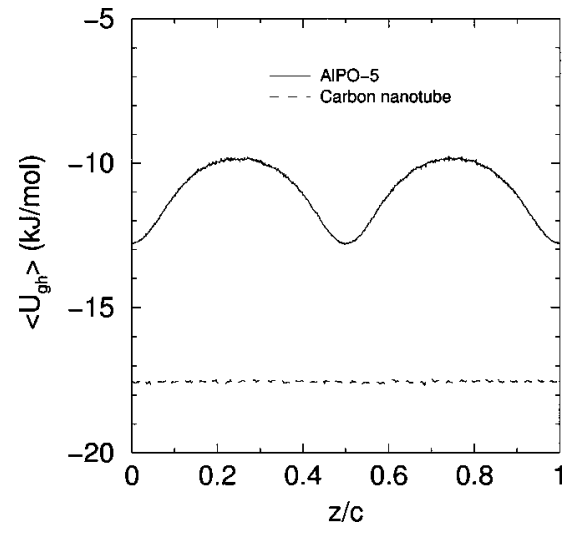

FIG. 2. Variation of $\left\langle U_{g h}\right\rangle$ as a function of the $z$ coordinate of the centerof-mass of methane at $T=200 \mathrm{~K} . z$ is scaled by the unit cell length of $\mathrm{AlPO}_{4}-5$ along the $c$-direction and by $49.294 \AA$ for the carbon nanotube.

phase the scaling of velocities is performed. This is followed by a production run of $150 \mathrm{ps}$. All runs have been carried out at a temperature of $200 \mathrm{~K}$.

For the methane molecule, the $\mathrm{C}-\mathrm{H}$ bond length is taken to be $1.1 \AA$. The mass of $\mathrm{C}$ and $\mathrm{H}$ is taken to be 12.01 and 1.008 amu. In what follows with rotation MD is termed as simulation $A$ and without rotation $\mathrm{MD}$ is termed as simulation $B$.

\section{RESULTS AND DISCUSSION}

\section{A. Energetics and structure}

This section contains results from the calculations of simulation $A$. The energetics and structure related results did not appear to be effected significantly by freezing of rotational degrees of freedom.

Figure 2 shows the average methane-host interaction energy $\left\langle U_{g h}\right\rangle$ as a function of the $z$-coordinate of the centerof-mass of methane over one unit cell length along the $c$-axis. Note that in all figures, $z$ is shown scaled by $c$, the unit cell length along the $c$ axis for respective channels. In $\mathrm{AlPO}_{4}-5$ there are peaks at $z / c \approx 0.25$ and 0.75 with a barrier of height $\approx 3 \mathrm{~kJ} / \mathrm{mol}$. The positions of peaks correspond to a region near the constriction of the channel in a unit cell of $\mathrm{AlPO}_{4}$-5. In the carbon nanotube there are no significant variations in $\left\langle U_{g h}\right\rangle$ as a function of $z$. This shows that in the narrow part of the channel in $\mathrm{AlPO}_{4}-5$ the repulsive part of the potential contributes more to $\left\langle U_{g h}\right\rangle$ than in the wider part of the channel. While in the carbon nanotube the contribution of the repulsive and dispersive part of the interaction energy remains almost constant along the channel.

Figure 3 shows a plot of $\left\langle U_{g h}\right\rangle$ as a function of $r$, the distance of the center-of-mass of methane from the channel axis. Note that in all figures $r$ is scaled by $R$, the radius of the respective channel defined from the van der Waals' free diameter. They are listed in Table II. In Table II, $d_{w}$ is obtained by subtracting $\sigma_{\mathrm{O}-\mathrm{O}}=2.529 \AA$ and $\sigma_{\mathrm{C}_{n}-\mathrm{C}_{n}}=3.4 \AA$ from $\sigma_{w}$ values of $\mathrm{AlPO}_{4}-5$ and the carbon nanotube, respectively. In the case of $\mathrm{AlPO}_{4}-5$ a simple arithmetic average over both narrow and wide regions yielded $R$. The radius values are 4.08 and $3.7 \AA$ for $\mathrm{AlPO}_{4}-5$ and the carbon nanotube, re- 


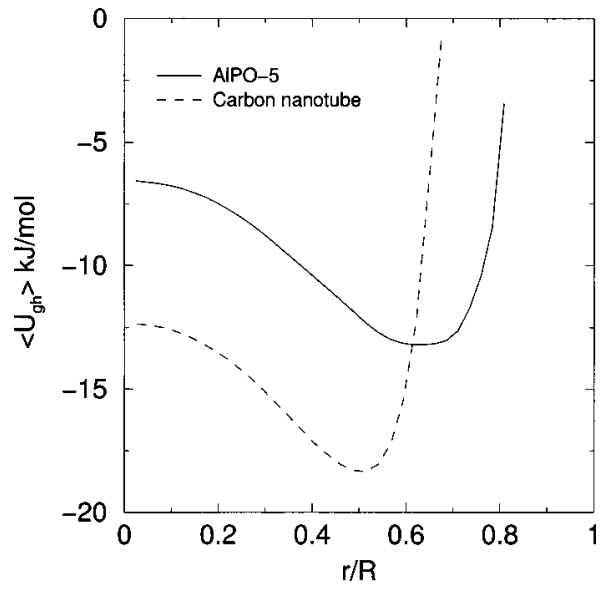

FIG. 3. Variation of $\left\langle U_{g h}\right\rangle$ as a function of $r$ perpendicular distance of center-of-mass of methane from the channel axis at $T=200 \mathrm{~K} . r$ is scaled by the radius $R$ of the respective channel.

spectively. As can be seen from Fig. 3, the minimum in the methane-host interaction is near the wall of the channel for both hosts. Table III lists the heat of adsorption $\Delta H_{\text {ads }}$ values calculated from $\Delta H_{\mathrm{ads}}=\left\langle U_{g h}\right\rangle-R T$, where $R$ is the gas constant and temperature $T=200 \mathrm{~K}$. Experimentally determined heat of adsorption values are listed in parentheses in Table III. The experimental $\Delta H_{\text {ads }}$ value was determined over the temperature range of 303-473 $\mathrm{K}$ for $\mathrm{AlPO}_{4}-5$ (Ref. 5) and $78-110 \mathrm{~K},{ }^{6} 155-195 \mathrm{~K}$ (Ref. 7) for the carbon nanotube. It can be seen from the table that calculated values are close to the experimentally determined values of heat of adsorption. It should be noted that experiments of methane adsorption on nanotubes were performed on tubes that were capped at both ends. Hence, methane adsorption takes place on the external surface of the nanotube. These values are tabulated for comparison and need not represent adsorption of methane inside a $(8,8)$ nanotube which is studied in the present work. Table III also lists $\left\langle U_{g g}\right\rangle$ values which are much smaller than the corresponding $\left\langle U_{g h}\right\rangle$ values.

Orientation of methane with respect to the channel axis is described by calculating the angle that each of the $\mathrm{C}-\mathrm{H}$ bonds $\left(C_{3}\right.$ axes) subtends to the channel axis. The minimum of these four angles, $\theta_{\min }$, is found out. The tetrahedral symmetry of the molecule limits the range of variation of $\theta_{\text {min }}$ between 0 to $\theta_{T_{d}} / 2$, where $\theta_{T_{d}}$ is the tetrahedral angle. $\theta_{\text {min }}$ helps in identifying two orientations of methane with respect to the channel axis. When $\theta_{\min }=\theta_{T_{d}} / 2$ or a $C_{2}$ axis of methane is parallel to channel axis, we term this orientation as perfect $2+2$ orientation and when $\theta_{\min }=0$ or a $C_{3}$ axis parallel to the channel axis we term this orientation as a perfect $1+3$ orientation. In a perfect $2+2$ orientation two of the

TABLE II. Dimension of the narrowest and the widest part of the host channel.

\begin{tabular}{lcc}
\hline \hline & $\sigma_{w}(\AA)$ & $d_{w}=\sigma_{w}-\sigma_{\text {host }}(\AA)$ \\
\hline $\mathrm{AlPO}_{4}-5$ & 10.02 & 7.49 \\
& 11.37 & 8.84 \\
Carbon nanotube & 10.80 & 7.4 \\
\hline \hline
\end{tabular}

TABLE III. Average interaction energy.

\begin{tabular}{lcc}
\hline \hline & $\Delta H_{\text {ads }}(\mathrm{kJ} / \mathrm{mol})$ & $\left\langle U_{g g}\right\rangle(\mathrm{kJ} / \mathrm{mol})$ \\
\hline $\mathrm{AlPO}_{4}-5$ & $-13.34\left(-14.3^{\mathrm{a}}\right)$ & -0.074 \\
Carbon nanotube & $-19.19\left(-18.3^{\mathrm{b}}-21.4^{\mathrm{c}}\right)$ & -0.086 \\
Bulk methane & & -4.1 \\
\hline \hline
\end{tabular}

${ }^{\mathrm{a}}$ Reference 5 .

${ }^{\mathrm{b}}$ Reference 6.

${ }^{\mathrm{c}}$ Reference 7.

$\mathrm{C}-\mathrm{H}$ bonds are symmetrical with respect to the channel axis and in a perfect $1+3$ orientation one of the $\mathrm{C}-\mathrm{H}$ bonds is parallel to the channel axis. A schematic representation of $1+3$ and $2+2$ orientations of methane with respect to a channel are as shown in Fig. 4. Orientations were classified as $1+3$, intermediate and $2+2$ if $0<\theta_{\min }<\theta_{T_{d}} / 6, \theta_{T_{d}} / 6$ $<\theta_{\min }<\theta_{T_{d}} / 3$ and $\theta_{T_{d}} / 3<\theta_{\min }<\theta_{T_{d}} / 2$, respectively. Any methane molecule inside the channels is in either of these three orientations.

We have calculated the number of methane molecules in each of the above orientations $n(z)$ and $n(r)$, where $z$ is the position of the center-of-mass of methane along the channel and $r$ is the radial distance of the center-of-mass of methane from the channel axis. In Figs. 5 and 6 the ratios $n(z) / N(z)$ and $n(r) / N(r)$ are plotted. $N(z)$ is the total number of methane molecules having their center-of-mass at $z$ along the channel and $N(r)$ is the total number of methane molecules having their center-of-mass at a distance $r$ from the channel axis. Figure 5 shows that the intermediate state is the most populated state at all values of $z$ in both $\mathrm{AlPO}_{4}-5$ and the carbon nanotube. In the carbon nanotube the $2+2$ state is dominating over $1+3$ throughout the length of the channel. However, in $\mathrm{AlPO}_{4}-5$ the modulation of the diameter of the channel gives rise to contrasting behavior. At the narrow parts of the channel, $z / c \approx 0.25$ and 0.75 , the $2+2$ state is more populated than the $1+3$ state, while at wider parts of the channel it is the $1+3$ state that is more populated than the $2+2$ state.

Figure 6 shows that the intermediate state of the orientation of methane is most populated among all orientation states for all values of $r$ for both hosts. In the carbon nanotube the $2+2$ state is populated more as compared to the $1+3$ state for all values of $r$. In $\mathrm{AlPO}_{4}-5$ near the center of the channel the $2+2$ state is populated more than the $1+3$ state while near the wall of the channel it is the $1+3$ state that is populated more than the $2+2$ state.

The above results suggest that there exists a correlation between channel diameter and the orientation of methane with respect to the channel axis. Table IV lists the diameter

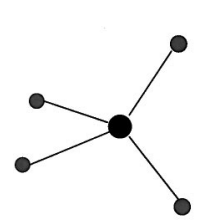

(a)

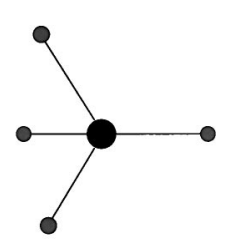

(b)
FIG. 4. A schematic representation of orientation of methane with respect to the channel in the (a) $2+2$ state and (b) $1+3$ state. 

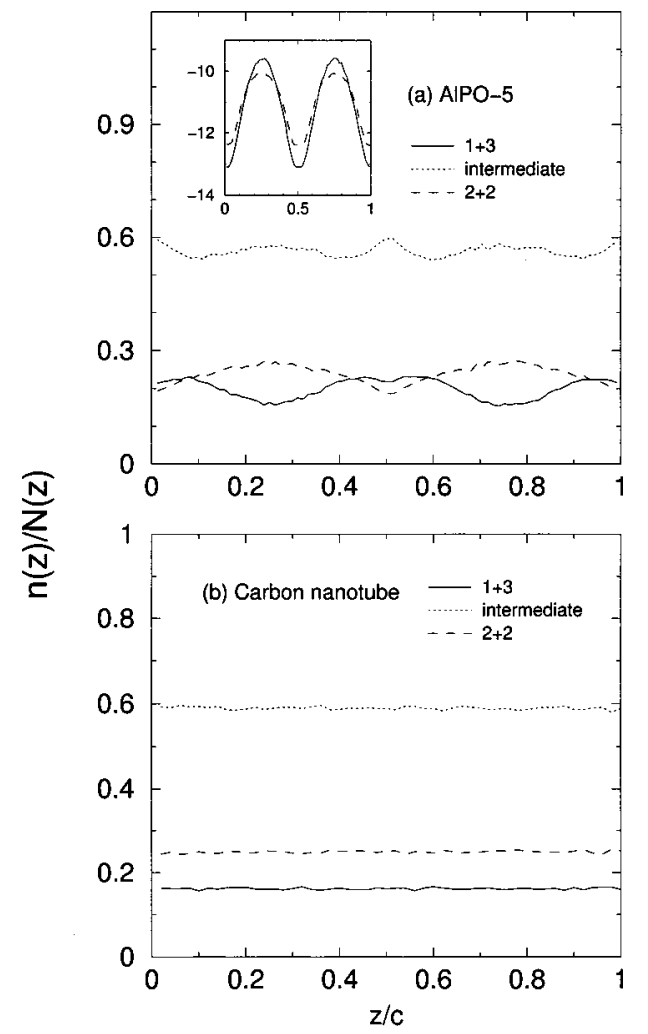

FIG. 5. The ratio $n(z) / N(z)$ as a function of position of the center-of-mass of methane along the channel. $n(z)$ is the number of methane molecules in a given state of orientation at $z$ and $N(z)$ are the total number of molecules at $z$. Inset in (a) shows the variation of $\left\langle U_{g h}\right\rangle$, in units of $\mathrm{kJ} / \mathrm{mol}$, with respect to $z$ for the $(1+3)$ and $(2+2)$ orientation. $z$ is scaled by the unit cell length $c$ for $\mathrm{AlPO}_{4}-5$ and by $c=49.294 \AA$ in the case of the carbon nanotube.

of the channel in $\mathrm{AlPO}_{4}-5$ and the carbon nanotube along with the levitation ratio ${ }^{21}$ defined as

$$
\gamma=\frac{2\left(2^{1 / 6} \sigma_{g h}+l\right)}{\sigma_{w}}
$$

where $\sigma_{g h}$ is the hydrogen-host interaction parameter, $\sigma_{w}$ is the window diameter defined as the distance between centers of diagonal atoms of a channel, and $l$ is the $\mathrm{C}-\mathrm{H}$ bond length. From Table IV it is clear that for values of $\gamma$ close to 1 (in close fitting parts of $\mathrm{AlPO}_{4}-5$ or in narrow channel-like carbon nanotube) more methane molecules have $2+2$ orientation than $1+3$ orientation. Similarly, in an earlier study of methane in $\mathrm{NaCaA},{ }^{22}$ it was observed that methane assumes $2+2$ orientation at the 8-ring window connecting two cages as it passes from one cage to another. The present study suggests under what conditions $(\gamma \rightarrow 1)$ a preference will be observed in the orientation of tetrahedral molecules. Further, earlier work on benzene in one-dimensional channels suggests that anisotropy in rotational diffusivity observed in the bulk is likely to be reversed in confinement when $\gamma \rightarrow 1 .^{23}$ These results when viewed together suggest that the levitation effect can alter drastically not only translational motion but also rotational motion.

The methane-host interaction energy for the $2+2$ and $1+3$ orientation as a function of $z$ and $r$ and averaged over the whole MD trajectory, are shown as insets in Figs. 5 and
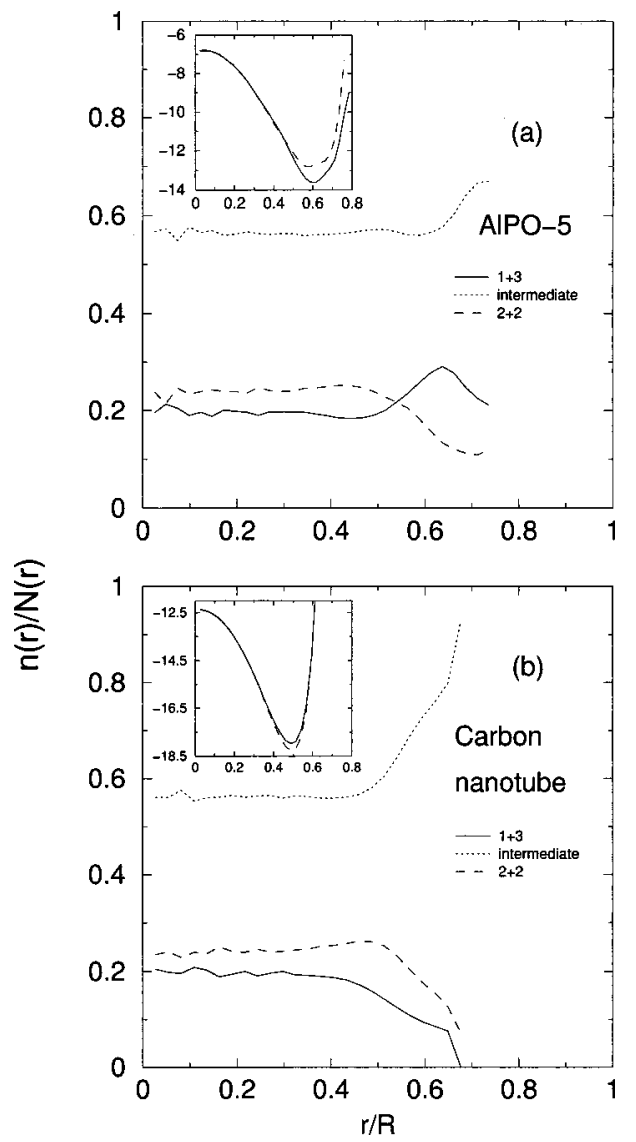

FIG. 6. The ratio $n(r) / N(r)$ as a function of radial distance of the centerof-mass of methane from the channel axis $r . n(r)$ is the number of methane molecules in a given state of orientation at $r$ and $N(r)$ are the total number of molecules at $r$. Inset in (a) and (b) shows the variation of $\left\langle U_{g h}\right\rangle$, in units of $\mathrm{kJ} / \mathrm{mol}$, with respect to $r$ for $(1+3)$ and $(2+2)$ orientations. $r$ is scaled by the radius $R$ of the corresponding channel.

6, respectively. The inset to Fig. 5 shows that in the narrow part of the $\mathrm{AlPO}_{4}-5$ channel repulsive contribution to the methane-host interaction energy in $1+3$ orientation is more than that in the $2+2$ orientation. While in the wider part of the channel in $\mathrm{AlPO}_{4}-5$ methane can maximize its interaction better in $1+3$ orientation than in $2+2$ orientation. As a function of the radial distance from the axis of the channel, $r$ (see inset to Fig. 6) methane maximizes its interaction with the host near the wall of the channel in $1+3$ orientation in $\mathrm{AlPO}_{4}-5$ and in $2+2$ orientation in the carbon nanotube.

Figure 7 shows the average value of the radial distance of the center-of-mass of methane from the channel axis as a function of its position along the channel. It can be seen that in $\mathrm{AlPO}_{4}-5$ methane is closer to the wall in wider parts of the channel and nearer to the center of the channel in narrow parts of the channel. In the carbon nanotube the average

TABLE IV. Dimension of the narrowest and the widest part of the host channel.

\begin{tabular}{lccc}
\hline \hline & $\sigma_{w}(\AA)$ & $2\left(2{ }^{1 / 6} \sigma_{g h}+l\right)(\AA)$ & $\gamma$ \\
\hline $\mathrm{AlPO}_{4}-5$ & 10.02 & 8.196 & 0.817 \\
Carbon nanotube & 11.37 & 8.196 & 0.720 \\
\hline \hline
\end{tabular}




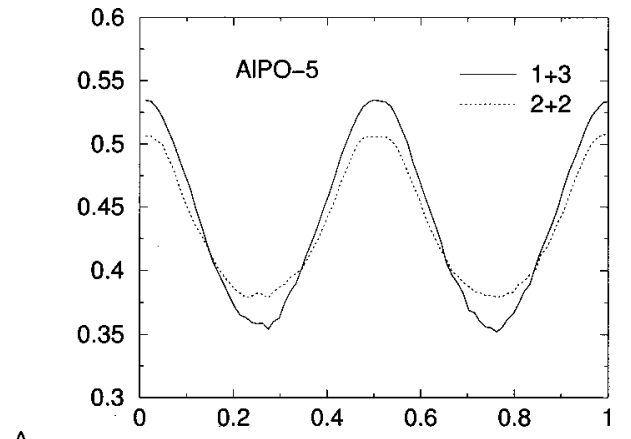

$\hat{\stackrel{\Upsilon}{\bar{v}}}$

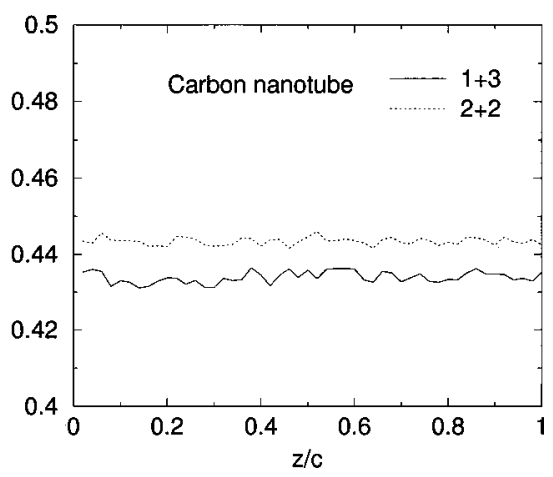

FIG. 7. The average radial distance of the center-of-mass of methane $\langle r\rangle$ from the channel axis as a function of position of center-of-mass of methane along the channel $z$. $\langle r\rangle$ vs $z$ is plotted for $1+3$ and $2+2$ orientations of methane. $r$ is scaled by the radius of the respective channel and $z$ is scaled by the unit cell length $c$ in $\mathrm{AlPO}_{4}-5$ and by $c=49.294 \AA$ in case of the carbon nanotube.

radial distance $\langle r\rangle$ of each of the orientations of methane remains almost constant along the channel. Among the two orientations, in the carbon nanotube, methane in the $2+2$ orientation is farther from the channel axis than the $1+3$ orientation. In $\mathrm{AlPO}_{4}-5$, in the narrow part of the channel the same behavior as the carbon nanotube is seen. The behavior is reversed when the channel diameter increases in the wider part of the channel; $2+2$ orientation is closer to the channel axis than the $1+3$ orientation.

These results suggest that the orientation of methane inside a one-dimensional channel is sensitive to the channel diameter. In these simulations the Lennard-Jones parameter $\sigma$ of the host atom determines the diameter of the channel. Pellenq and Nicholson ${ }^{24}$ recently made an accurate determination of Lennard-Jones parameters between rare gases and silicalite-1 host atoms. Similar studies are desirable for hydrocarbon-zeolite interactions. We hope that results here provide a sensitive and crucial test of these parameters. Experiments which can distinguish between $2+2$ and $1+3$ orientations will be able to yield valuable insight into the accuracy of parameters between $\mathrm{CH}_{4}$ and zeolite.

\section{B. Dynamical properties}

\section{Translational motion}

Figure 8 shows the mean squared displacement of methane in $\mathrm{AlPO}_{4}-5$ and the carbon nanotube and in bulk, for simulation $A$ and simulation $B$. The mean squared displacement of methane varies linearly with time for bulk case and in $\mathrm{AlPO}_{4}-5$. But in the case of the carbon nanotube it does
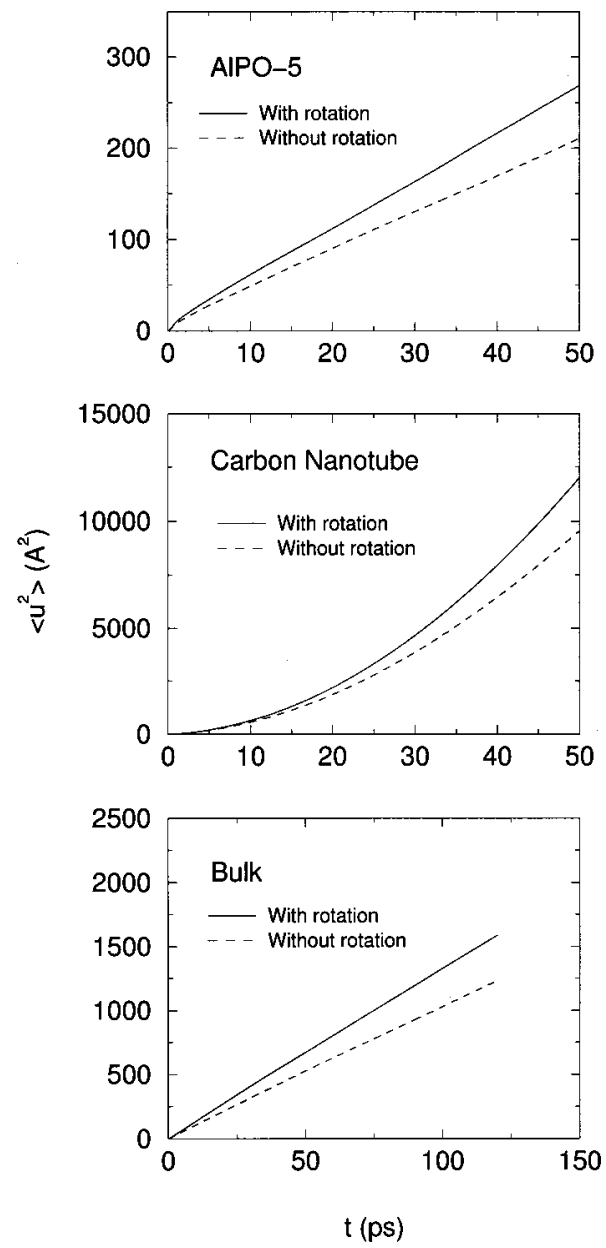

FIG. 8. Mean squared displacement of methane in $\mathrm{AlPO}_{4}-5$, carbon nanotube, and bulk for the case of simulation $A$ and simulation $B$ at $T=200 \mathrm{~K}$.

not go over to the diffusive regime. The exponent $\alpha$ in the relation $\left\langle u^{2}(t)\right\rangle \approx t^{\alpha}$ can be obtained from the log-log plot. For methane in the carbon nanotube $\alpha=1.811$ and 1.749 for simulation $A$ and simulation $B$, respectively. $\alpha=1$ corresponds to the case of diffusive motion while $\alpha=2$ corresponds to the ballistic or free particle motion. Methane motion along the $z$-direction in the nanotube, unlike in $\mathrm{AlPO}_{4}-5$, experiences almost uniform potential energy surface along the channel (see Fig. 2) which leads to nearly free particlelike motion. When this is coupled with nonballistic motion along radial directions, one obtains a value between 1 and 2 for the exponent $\alpha$. The motion is expected to go over to the diffusive regime as the number of methane molecules per tube are increased. ${ }^{15}$

The self-diffusion coefficient is calculated in the case of bulk methane and methane in $\mathrm{AlPO}_{4}-5$ by Einstein's relation,

$$
D^{t}=\frac{\left\langle r^{2}\right\rangle}{6 t} .
$$

The $D^{t}$ values are reported in Table $\mathrm{V}$. The straight line is fitted over the range of $10-40 \mathrm{ps}$. The error bars in $D^{t}$ for methane in $\mathrm{AlPO}_{4}-5$ are $2.7 \%$ and $2.2 \%$ for the case of simulation $A$ and simulation $B$, respectively. The error bars for bulk methane self-diffusivity are $1.3 \%$ and $2.4 \%$ for the 
TABLE V. Self-diffusion coefficient.

\begin{tabular}{lccc}
\hline \hline & $\begin{array}{c}D^{t}, \text { simulation } A \\
\left(10^{-8} \mathrm{~m}^{2} / \mathrm{s}\right)\end{array}$ & $\begin{array}{c}D^{t}, \text { simulation } B \\
\left(10^{-8} \mathrm{~m}^{2} / \mathrm{s}\right)\end{array}$ & $\begin{array}{c}\text { density, } \rho \\
\mathrm{g} / \mathrm{cm}^{3}\end{array}$ \\
\hline $\mathrm{AlPO}_{4}-5$ & 0.867 & 0.669 & 0.02 \\
Bulk methane & 2.23 & 1.74 & 0.29 \\
\hline \hline
\end{tabular}

case of simulation $A$ and simulation $B$, respectively. As can be seen from Fig. 8 and Table $\mathrm{V}$ there is a difference in mobility of methane for the two cases of simulation $A$ and simulation $B$. Methane is less mobile in simulation $B$ in all the three systems studied. This indicates that rotation enhances translational motion of methane in the bulk state as well as in confined systems like $\mathrm{AlPO}_{4}-5$ and the carbon nanotube. Self-diffusion coefficient $D^{t}$, in general, depends on the density of sorbates inside a porous host. ${ }^{1}$ It is generally expected that with the increase in density the value of $D^{t}$ decreases. Table $\mathrm{V}$ indicates that even though density of methane is lower in the channels of $\mathrm{AlPO}_{4}-5$ than the bulk density, the diffusivity of methane is also lower than the bulk methane diffusivity value. This reduction in mobility can be due to the presence of constrictions in $\mathrm{AlPO}_{4}-5$ which hinder the motion of methane along the channel.

The motion of methane in simulation $B$ in $\mathrm{AlPO}_{4}-5$ and the carbon nanotube is further analyzed by considering the mean squared displacement of each molecule separately. This provides information about the relationship between the mobility of a molecule and its orientation. This is easier for simulation $B$ as each molecule has a different orientation which does not change throughout the MD run. Figures 9

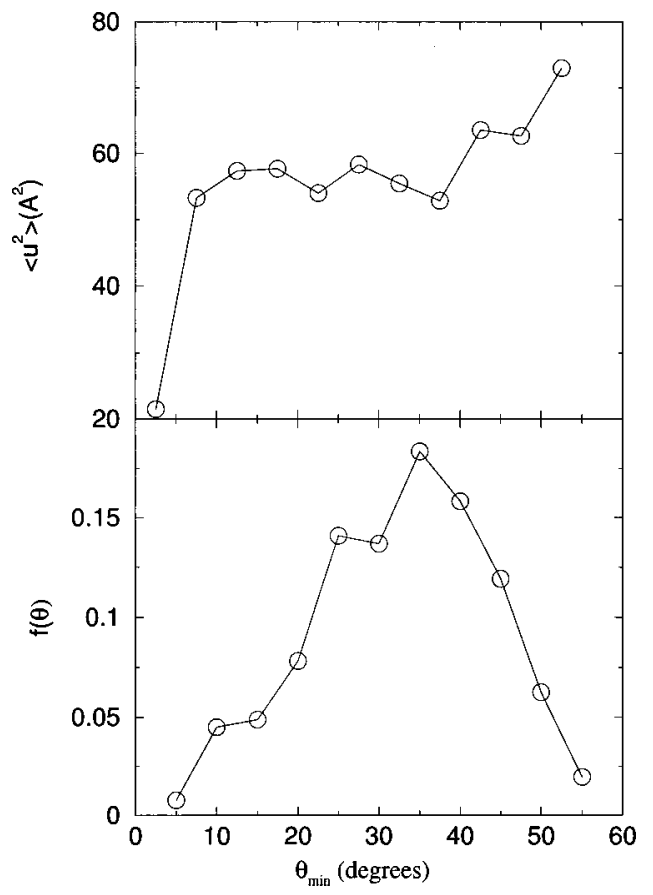

FIG. 9. For the $\mathrm{AlPO}_{4}-5$ value of the mean squared displacement at the end of $12 \mathrm{ps,} \mathrm{calculated} \mathrm{for} \mathrm{each} \mathrm{methane} \mathrm{molecule} \mathrm{in} \mathrm{simulation} B$ and averaged over molecules that have their $\theta_{\min }$ values in a given interval of width $5^{\circ}$. Also plotted is the distribution of methane molecules over $\theta_{\min }$ by dividing the range into intervals of width $5^{\circ}$.

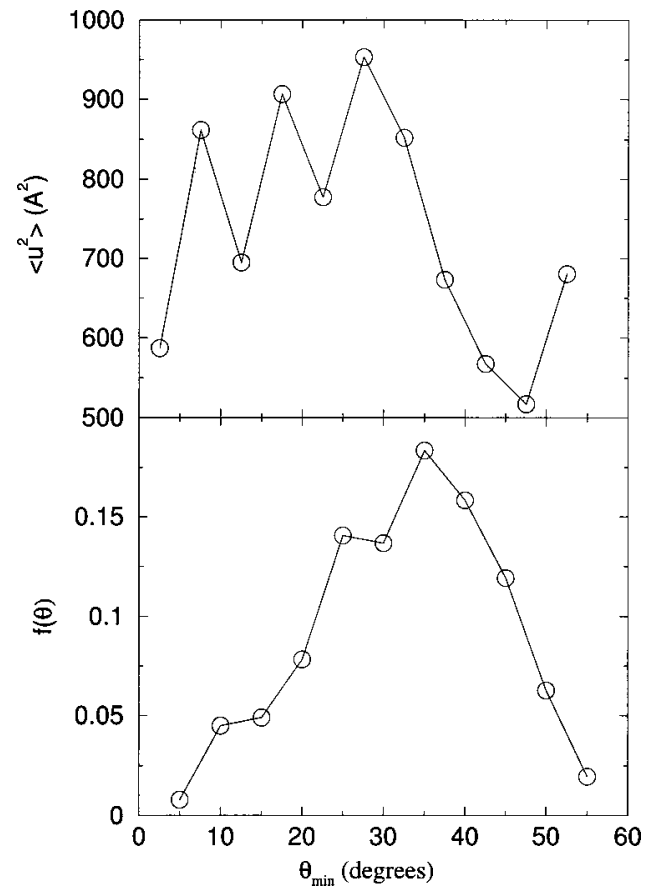

FIG. 10. For the carbon nanotube value of the mean squared displacement at the end of $12 \mathrm{ps,} \mathrm{calculated} \mathrm{for} \mathrm{each} \mathrm{methane} \mathrm{molecule} \mathrm{in} \mathrm{simulation} B$ and averaged over molecules that have their $\theta_{\min }$ values in a given interval of width $5^{\circ}$. Also plotted is the distribution of methane molecules over $\theta_{\min }$ by dividing the range into intervals of width $5^{\circ}$.

and 10 show the plot of the mean squared displacement as a function of $\theta_{\min }$ and the distribution function of $\theta_{\min }$ for $\mathrm{AlPO}_{4}-5$ and the carbon nanotube, respectively. The distribution function is calculated by dividing the range 0 to $\theta_{T_{d}} / 2$ into intervals each of $5^{\circ}$ width. The number of methane molecules in each of these intervals are calculated. For each of the methane molecules in a given interval mean squared displacement is calculated for $12 \mathrm{ps}$. The value of mean squared displacement at the end of $12 \mathrm{ps}$ is averaged over all molecules in that interval and is reported here. These values of $\left\langle u^{2}\right\rangle$ are plotted in Figs. 9 and 10 as a function of $\theta_{\min }$.

The definition of the three states of orientation of methane, as defined earlier, can be used to analyze the results from Figs. 9 and 10. Figures 9 and 10 indicate that the intermediate state of orientation for methane contributes substantially to the translational mobility in $\mathrm{AlPO}_{4}-5$ and the carbon nanotube. In $\mathrm{AlPO}_{4}-5$, the $1+3$ state does not contribute significantly to the mobility for $\theta_{\min }<10^{\circ}$. The $2+2$ state contributes significantly to translational mobility as compared to other orientations of methane in $\mathrm{AlPO}_{4}$-5. In contrast, the carbon nanotube $2+2$ state does not have significant contribution to mobility as compared to other states of orientation of methane.

The $x$ - and $y$-components of the velocity autocorrelation function for methane in $\mathrm{AlPO}_{4}-5$ and the carbon nanotube decay in the expected manner (not shown). The $z$-component of methane in $\mathrm{AlPO}_{4}-5$ decays to zero. In the carbon nanotube this does not happen. Therefore, the mean squared displacement exhibits nondiffusive behavior. 
TABLE VI. Rotational diffusivity of methane.

\begin{tabular}{lccc}
\hline \hline & $D_{x}^{r}\left(\mathrm{ps}^{-1}\right)$ & $D_{y}^{r}\left(\mathrm{ps}^{-1}\right)$ & $D_{z}^{r}\left(\mathrm{ps}^{-1}\right)$ \\
\hline AlPO $_{4}-\mathbf{5}$ & & & \\
Body-fixed frame & 16.26 & 16.38 & 16.38 \\
Space-fixed frame & 4.69 & 4.77 & 4.3 \\
Carbon nanotube & & & \\
Body-fixed frame & 25.99 & 26.99 & 27.04 \\
Space-fixed frame & 5.62 & 6.08 & 7.32 \\
Bulk methane & & & 9.85 \\
Body-fixed frame & 9.04 & 8.86 & \\
\hline \hline
\end{tabular}

\section{Rotational motion}

The angular velocity correlation function calculated in the body-fixed frame of methane (data not shown) indicated that correlations in angular velocity components decay in identical fashion for bulk as well as confined methane. The difference is in the time the correlations take to decay to zero for each system. This is reflected in the rotational diffusivity calculated by,

$$
D_{i}^{r}=\int_{0}^{\infty}\left\langle\omega_{i}(0) \omega_{i}(t)\right\rangle d t,
$$

where $i=x, y, z$. The integration is performed up to $10 \mathrm{ps}$. The rotational diffusivity calculated is listed in Table VI. The rotational diffusivity values indicate that methane rotates faster in confinement which corresponds to low density as compared to bulk (at $\rho=0.29 \mathrm{~g} / \mathrm{cm}^{3}$ ) which is as expected. And in confinement it rotates faster in the carbon nanotube than in $\mathrm{AlPO}_{4}-5$. Angular velocity autocorrelation function can be calculated in space fixed frame also. This is plotted in

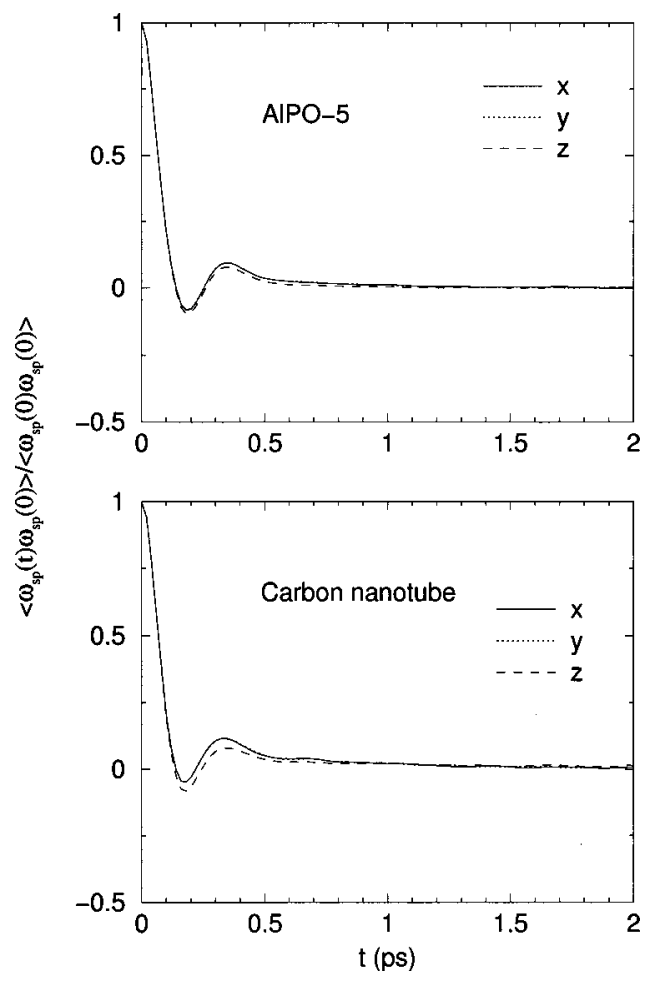

FIG. 11. Angular velocity autocorrelation function for methane in the spacefixed frame in $\mathrm{AlPO}_{4}-5$, carbon nanotube and bulk at $T=200 \mathrm{~K}$.
Fig. 11. Angular velocity in the space-fixed frame gives information about the rotation of the molecule about the space fixed directions. From an integration of angular velocity autocorrelation function in the space-fixed frame one can obtain rotational diffusivities. These values are listed in Table VI. From the table it can be seen that in $\mathrm{AlPO}_{4}-5$ and in the carbon nanotube there exists a significant amount of rotational motion around all the space fixed axes.

\section{CONCLUSIONS}

The properties of methane are strongly dependent on the nature of the one-dimensional channel as well as on the ratio of size of the molecule to the channel diameter. The orientation of methane with respect to the channel axis shows interesting properties in $\mathrm{AlPO}_{4}$-5. In narrow parts of the channel in $\mathrm{AlPO}_{4}-5$ the orientation in which a $C_{2}$ axis of methane is directing along the channel axis $(2+2$ orientation) is preferred over the orientation in which a $C_{3}$ axis of methane is directed along the channel axis $(1+3$ orientation) and vice versa in the wider part of the channel. This preference for a particular orientation is also seen when methane is closer to the channel wall in $\mathrm{AlPO}_{4}$-5. In the wider region, near the channel wall, methane assumes a $1+3$ orientation while in narrow regions the $2+2$ orientation is preferred by methane when it gets closer to the channel wall.

The effect of correlation between orientation and position of methane inside a channel extends to dynamical properties as well. It is seen that the mean square displacement of methane as probed over the first 12 ps depends strongly on its orientation. In $\mathrm{AlPO}_{4}-5$, it is seen that when $\theta_{\text {min }}<10^{\circ}$, $u^{2}(t)$ is small and in the carbon nanotube, large values of $\theta_{\min }$ have lower values of $u^{2}(t)$. These suggest the strong dependence of translational mobility on the orientation of methane. It follows that a single site model of methane may not be able to reproduce all the properties of methane$\mathrm{AlPO}_{4}-5$ and methane-carbon nanotube system.

These results are consistent with our results of runs where diffusivities have been calculated with and without rotation; simulation $B$ and simulation $A$ show that rotation aids in translational motion and enhances translational diffusivity in bulk as well as in confinement. Note that methane shows nondiffusive behavior in the carbon nanotube. Since lattice vibrations in $\mathrm{AlPO}_{4}-5$ and the carbon nanotube are not expected to be large, we expect that the results presented here would not alter significantly when the host atoms are also included in the dynamics. It is also seen that rotational diffusivity of methane is the highest in carbon nanotube which has smooth channel walls as compared to rotational diffusivity of methane in $\mathrm{AlPO}_{4}-5$ and in bulk.

\section{ACKNOWLEDGMENTS}

Financial support from the Department of Science and Technology, New Delhi, is gratefully acknowledged. S.Y.B. thanks CSIR, New Delhi, for the award of a research fellowship. 
${ }^{1}$ J. Kärger and D. M. Ruthven, Diffusion in Zeolites and Other Microporous Solids (Wiley, New York, 1992).

${ }^{2}$ S. P. Bates and R. A. van Santen, Adv. Catal. 42, 1 (1997).

${ }^{3}$ L. A. Clark, G. T. Ye, A. Gupta, L. L. Hall, and R. Q. Snurr, J. Chem. Phys. 111, 1209 (1999).

${ }^{4}$ L. N. Gergidis, D. N. Theodorou, and H. Jobic, J. Phys. Chem. B 104, 5541 (2000).

${ }^{5}$ V. R. Choudhary and S. Mayadevi, Sep. Sci. Technol. 28, 2197 (1993).

${ }^{6}$ M. Muris, N. Dufau, M. Bienfait, N. Dupont-Pavlovsky, Y. Grillet, and J.

P. Palmari, Langmuir 16, 7019 (2000).

${ }^{7}$ S. E. Weber, S. Talapatra, C. Journet, A. Zambano, and A. D. Migone, Phys. Rev. B 61, 13150 (2000).

${ }^{8}$ V. Lachet, A. Boutin, R. J. M. Pellenq, D. Nicholson, and A. Fuchs, J. Phys. Chem. 100, 9006 (1996).

${ }^{9}$ T. Maris, T. J. H. Vlugt, and B. Smit, J. Phys. Chem. B 102, 7183 (1998).

${ }^{10}$ V. Kukla, J. Kornatowski, D. Demuth, I. Girnus, H. Pfeiffer, L. Rees, S. Schunk, K. Unger, and J. Kärger, Science 272, 702 (1996).

${ }^{11}$ S. S. Nirvathi, A. V. McCormick, and H. T. Davis, Chem. Phys. Lett. 229, 297 (1994).
${ }^{12}$ H. Jobic, H. Hahn, J. Kärger, M. Bée, A. Tuel, M. Noack, I. Girnus, and J. K. Gordon, J. Phys. Chem. B 101, 5834 (1997).

${ }^{13}$ K. T. Thomson, A. V. McCormick, and H. Ted Davis, J. Chem. Phys. 112, 3345 (2000).

${ }^{14}$ D. S. Sholl, Chem. Eng. J. 74, 25 (1999).

${ }^{15}$ Z. Mao and S. B. Sinnott, J. Phys. Chem. B 104, 4618 (2000).

${ }^{16}$ J. W. Richardson, Jr., J. J. Pluth, and J. V. Smith, Acta Crystallogr., Sect. C: Cryst. Struct. Commun. 43, 1469 (1987).

${ }^{17}$ K. G. Ayappa, Langmuir 14, 880 (1998).

${ }^{18}$ F. Zhang, J. Chem. Phys. 111, 9082 (1999).

${ }^{19}$ S. Murad and K. E. Gubbins, Computer Modeling of Matter, ACS Symposium Series, edited by P. Lykos (American Chemical Society, Washington, DC, 1978), Vol. 86, p. 62.

${ }^{20}$ M. P. Allen and D. J. Tildesley, Computer Simulation of Liquids (Clarendon, Oxford, 1987).

${ }^{21}$ S. Yashonath and P. Santikary, J. Phys. Chem. 98, 6368 (1994).

${ }^{22}$ R. Chitra, A. V. Anil Kumar, and S. Yashonath, J. Chem. Phys. 114, 11 (2001).

${ }^{23}$ S. Y. Bhide and S. Yashonath, J. Phys. Chem. B 104, 11977 (2000).

${ }^{24}$ R. J. Pellenq and D. Nicholson, J. Phys. Chem. 98, 13339 (1994). 